\title{
Altitude of pulsating arcs as inferred from tomographic measurements
}

Vladimir Safargaleev ${ }^{1}$, Tima Sergienko ${ }^{2 *}$ (1), Keisuke Hosokawa ${ }^{3}$, Shin-ichiro Oyama ${ }^{4,5,6}$, Yasunobu Ogawa ${ }^{5,7,8}$, Yoshizumi Miyoshi ${ }^{4}$, Satoshi Kurita ${ }^{9}$ and Ryochi Fujii ${ }^{10}$

\begin{abstract}
Data from three all-sky cameras in Kiruna and Tjautjas (Sweden) were used to estimate the altitude of pulsating arclike forms using optical tomography. The event under consideration occurred during the substorm recovery phase and comprised both periodic luminosity variation of the on/off type with repetition periods of 3-6s (main pulsations) and faster scintillation (approximately $2 \mathrm{~Hz}$ ) during the "on" phase of the main pulsations. It is found that (1) the altitudes of the pulsating auroral arcs decrease during "on" intervals from $~ 95 \mathrm{~km}$ to $\sim 92 \mathrm{~km}$ and (2) for two closely spaced arcs, internal modulation took place only in the lowest arc. The results may be interpreted in the frame of the traditional mechanism assuming electron scattering via VLF-wave/particle interaction in the equatorial magnetosphere, while the internal modulation may also be alternatively interpreted in the frame of the less-often inferred mechanism of field-aligned acceleration somewhere between the equatorial plane and ionosphere.
\end{abstract}

Keywords: Pulsating auroras, Internal modulation, Altitude of auroras, Optical tomography

\section{Graphical Abstract}

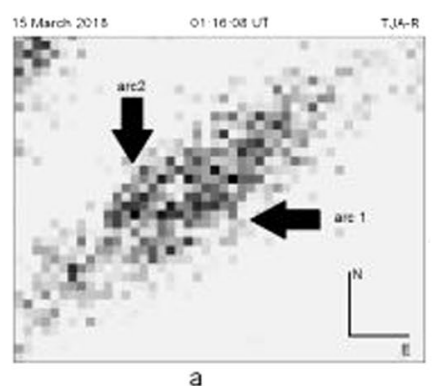

(a) two closely spaced arcs

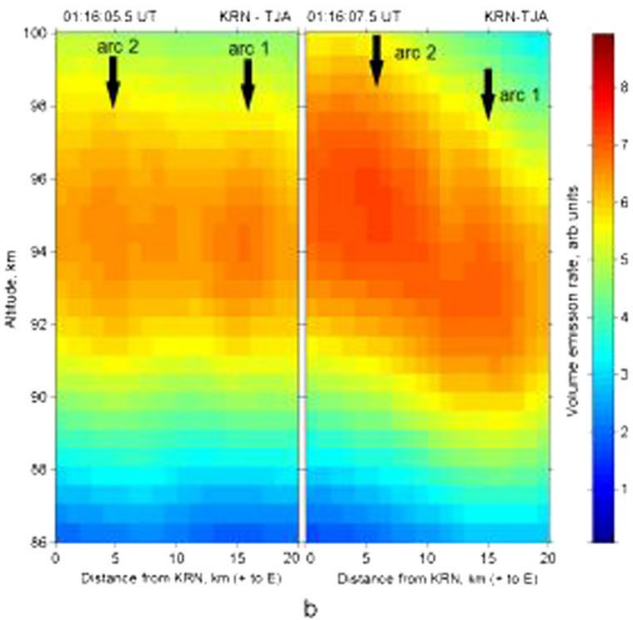

b

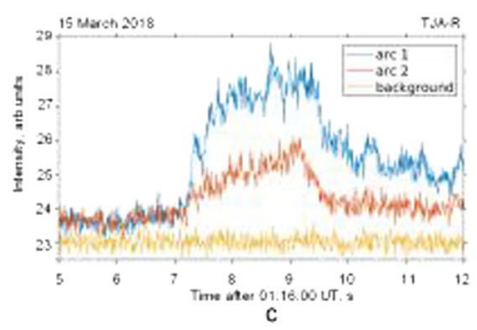

(c) internal modulation is seen in the lower arc 1

(b) altitude of the brightenning arc 1 decreases during the main pulse

*Correspondence: tima.sergienko@irf.se

2 Swedish Institute of Space Physics, Kuruna, Sweden

Full list of author information is available at the end of the article

\section{Main text}

\section{Introduction}

Pulsating aurora (PsA) is the name given to a kind of diffuse aurora that appears, in general, as irregular patches 
or more regular arc/segment structures with quasi-periodic on-off switching of its intensity. The term covers a wide range of auroras, which may arise from different processes and different source regions. Most of the known characteristics of PsA have been reviewed by Royrvik and Davis (1977), Johnstone (1978), Lessard (2012), Nishimura et al. (2020). In particular, Royrvik and Davis (1977) established three general morphologies: east-west aligned arcs, quasi-linear elements, and patches. The latter are regarded as the most common form of PsA and may also be divided into three subcategories (Grono and Donovan 2020). This study focuses on arc-like structures. Previous results closest to our research are outlined below.

Nishimura et al. (2020) pointed out seven types of structures with intensity modulation. One of the types includes $\sim 3-\mathrm{Hz}$ modulation. Recently, Hosokawa et al. (2020) used the term "beats" concerning the brightness of PsA, which are characterized by a mixture of two distinct periodicities that coexist hierarchically. One is the "main pulsations", which is the primary periodicity component ranging from a few to a few tens of seconds. The other is the so-called "internal modulation", which is quicker luminosity scintillation (a few $\mathrm{Hz}$ ) embedded in a single pulse of the main pulsations. The study by Royrvik and Davis (1977) showed that $3 \pm 1 \mathrm{~Hz}$ modulation appears in more than $50 \%$ of all PsA events that occur near the equatorward boundary of the auroral zone in the midnight and morning sector.

The interesting finding by Royrvik and Davis (1977) is that in many cases, $3 \pm 1 \mathrm{~Hz}$ modulation is detected only near the form boundary. Later, Sato et al. (2004) suggested that the $\sim 3 \mathrm{~Hz}$ luminosity oscillations in pulsating arc-like forms during substorm recovery are aligned along the arc rather than north-south, which is in agreement with the above result by Royrvik and Davis (1977). They also noted that, for the homogeneous form, the modulation is synchronous wherever it is observed in the form. If the forms show an internal structure, each element of the structure exhibits modulation, and the phase and exact frequency are independent of other elements. One should keep this in mind when analyzing all-sky images because the low spatial resolution often does not allow the fine structure of pulsating arc-like forms to be seen on the original image without special processing.

One area of substantial interest is the altitude of pulsating patterns and how it varies over the course of pattern evolution. This information may help in understanding the nature of PsA and can be inferred from ground observations. Triangulation of different auroral forms gave the altitude range of PsA from 82 to $105 \mathrm{~km}$ with a median altitude of $92 \mathrm{~km}$ (Brown et al. 1976), which is noticeably lower than that for post-breakup homogenous arc-like forms. Primary electron energy near $60 \mathrm{keV}$ is required to produce the lowest observed pulsating aurora. In addition, initial observations by Brown et al. (1976) showed no indication of changes in the height of PsA during the lifetime of a single pulsation. However, Kataoka et al. (2016) recently performed high-speed stereoscopy of the aurora, which is also based on the triangulation method, and found an increase in the emission altitude of both pulsating patches and arcs over the course of their development. This result seems to be unexpected because it implies a decrease in the energy of precipitating electrons causing luminosity enhancement, whereas rocket experiments suggest otherwise (e.g., McEwen et al. 1981). Whiter et al. (2010) used the data from two Aurora Structure and Kinetics cameras, each sensitive to different precipitating electron energies, and showed that, in the events studied, the energy of flickering electron precipitation was higher than the energy of the non-flickering auroral electrons. This may indicate that the altitude of the flickering aurora was lower than the altitude of non-flickering forms, with both arcs also demonstrating periodicities of a few seconds.

The effects of PsA on ionospheric electrodynamics are studied by using both ground-based and spacebased instruments. Using the EISCAT radar in Tromsø, Hosokawa et al. (2010) demonstrated that an escape of charges from the edges of pulsating aurora patches could be associated with field-aligned currents, FAC. Recently, Gillies et al. (2015) introduced observations of FACs in the vicinity of pulsating aurora patches using the Swarm satellite. They identified strong downward currents just poleward and equatorward of the pulsating patches, whereas weaker upward currents were observed throughout the interior of the patches.

Rocket and satellite measurements have shown that PsA is an optical manifestation of quasi-periodic modulation of the flux of precipitating high-energy electrons with energies from a few to a few tens of $\mathrm{keV}$ (Tesema et al. 2020; Nishimura et al. 2020 and references therein). In particular, Sandahl et al. (1980), Sato et al. (2004) and Miyoshi et al. (2015) reported quasi-3 Hz modulation in the downcoming electron fluxes corresponding to the main body of pulsations with periods of a few seconds that were detected onboard rocket and low-altitude satellites. McEwen et al. (1981) found that optical pulsations with an approximately $17-\mathrm{s}$ period are due to periodic increases in the energy of the electrons from $1.5 \mathrm{keV}$ during pulsation minima to approximately $1.8 \mathrm{keV}$ at pulsation maxima, and electron spectra have a Maxwellian energy distribution. Since no indications of local acceleration were found, McEwen et al. (1981) connected the observations of energy increase with adiabatic heating of the source electrons. Note that the results were obtained 
during two rocket launches and were related to a pulsating patch and some extended pulsating surface in the diffuse aurora with periodicity noticeably larger than the typical periodicity (a few seconds). Although the direct relationship of onboard measurements with groundbased optical observations was declared, no evidence of conjugation of the rockets with particular pulsating auroral forms was presented.

The auroral electron velocity dispersion measured during pulsations suggested the source of a few-second modulation to be in the vicinity of the geomagnetic equatorial plane (e.g., Bryant et al. 1971). The conclusion was based on rocket observations outside the ground photometer field of view. Therefore, neither the shape of the pulsating pattern nor its conjugation with the rocket could be established. Later, Sato et al. (2004) showed that the source region of generation or modulation of both $5 \mathrm{~s}$ and $\sim 3 \mathrm{~Hz}$ precipitation is located earthward, far from the equatorial plane, at $\sim 2-6 R_{E}$ above the Fast Auroral Snapshot Explorer (FAST) along the field line. The PsA consisted of arc-like forms, and its conjugation with the satellite was more obvious.

Pulsating aurora has been studied for decades, but its generation and modulation mechanisms are still open issues. On the one hand, the location of the source of PsAs in the equatorial plane and the absence of signs of electron acceleration suggest that electrons responsible for pulsating aurora are launched from the magnetosphere into the ionosphere by pitch-angle scattering. Candidate waves to scatter electrons are lower-band chorus (LBH) and electrostatic electron cyclotron harmonic $(\mathrm{ECH})$ waves. The results of a case study of groundArase satellite conjugation suggested that LBH cause short-period pulsating aurora at low altitudes, while ECH waves cause long-period PsA at high altitudes (Fukizawa et al. 2018). Later, a two-case study of Arase conjugation with ground-based optical instruments showed a one-toone correlation between chorus elements and the $\sim 3 \mathrm{~Hz}$ modulation of pulsating patterns (Hosokawa et al. 2020).

On the other hand, as noted by Sato et al. (2015), the model of periodic modulation of high-energy electron precipitation has mainly been applied to patch-type pulsating auroras. If one applies such a model to pulsating arcs, it may be very difficult to explain how physical processes can produce so greatly elongated structures. Signatures of electron acceleration were found in FAST data by Sato et al. (2004). A dispersion analysis places the source region of precipitating electrons between 2 and $6 \mathrm{RE}$ above FAST, which is far from the equatorial source region. They also show out-of-phase modulation of downgoing, high-energy ion and electron fluxes. Based on these results, they supposed that time variations of the field-aligned potential drop may cause the pulsating aurora. This result supports the earlier comment by Johnstone (1978), who noticed that the apparent vertical extent of the luminosity is less than $10 \mathrm{~km}$, whereas a Maxwellian electron spectrum should give a luminosity profile at least $30 \mathrm{~km}$ thick. Thus, along with periodic pitch-angle scattering, the modulation of field-aligned acceleration can also be regarded as a possible generation mechanism of PsA.

That differing theories of the mechanism of pulsating aurora still exist has at least two reasons. First, there are few in situ observations. Indeed, rocket and satellite studies represent limited case studies. Second, it is noteworthy that in many cited papers, there is no direct indication of exactly what kind of auroral form is under consideration (Bryant et al. 1971; Arnoldy et al. 1982; Miyoshi et al. 2015; Tesema et al. 2020), and the difference in pulsating forms is not emphasized. Instead, the common term "pulsating aurora" is often used. The morphology and nature of different pulsating forms may be different.

In this study, we focus on measurements of the altitude of pulsating arc-like forms with ground-based optical instruments and how the altitude varies over the course of pattern evolution. This information may help in understanding the nature of pulsating aurora since, for example, a decrease in altitude may indicate acceleration of the particles. The aurora height was estimated by means of optical tomography. The events considered were a very typical type of pulsating aurora because they occur during the recovery phase of magnetospheric substorms and demonstrate a few seconds and $\sim 2-3 \mathrm{~Hz}$ modulations coexisting hierarchically.

\section{Instrumentation and methods}

The data of three all-sky cameras were used in this study. The EMCCD all-sky camera in Tjautjas (TJA; $67.31^{\circ} \mathrm{N}$, $20.73^{\circ} \mathrm{E}$ ), Sweden, that was installed under the PsA research project and PWING project (e.g., Shiokawa et al. 2017) gives 100 images per second that allow us to detect both the main pulsations and the internal modulation in PsA. Two identical sets of all-sky Watec Monochromatic Imagers (WMI) of the National Institute of Polar Research, Japan, installed in Kiruna (KRN; $67.88^{\circ} \mathrm{N}$, $20.42^{\circ} \mathrm{E}$ ) and Tjautjas (see Fig. 1a) with a north-south base distance of $\sim 60 \mathrm{~km}$ give images with a time resolution of $1 \mathrm{~s}$; therefore, pulsating aurora with the period of the main pulsations can be targeted for study (Ogawa et al. 2020). To distinguish between PsA and WMI cameras, both installed in Tjautjas, we will further refer to the EMCCD camera in Tjautjas as a rapid or high-speed or TJA-R camera.

The images taken with the WMI cameras were used for the reconstruction of the volume distribution of 


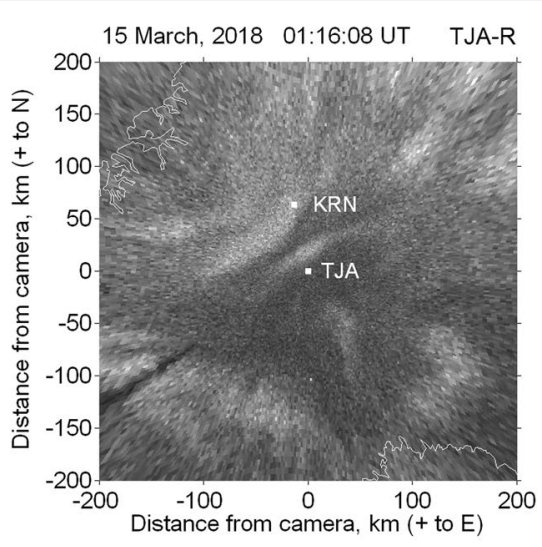

a

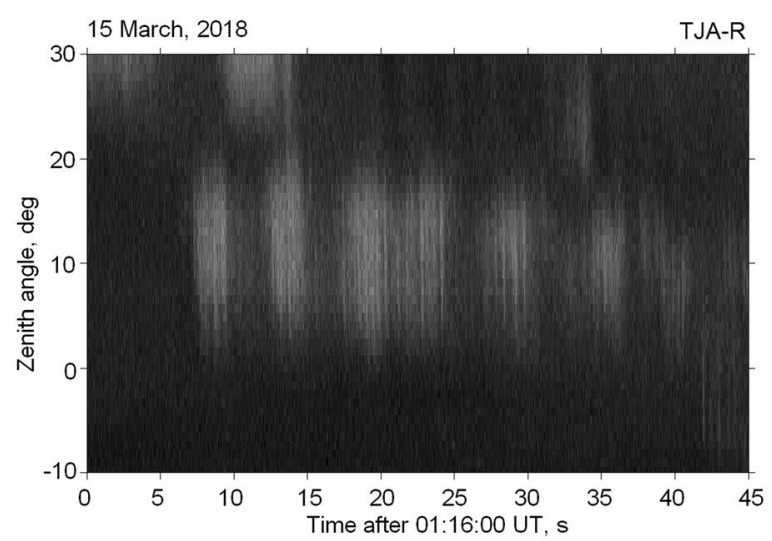

b

Fig. 1 a Location of pulsating (zenith) arc relative to the KRN and TJA observatories (white squares). b Keogram showing the temporal variation in auroral brightness sampled along the south to north cross section. A series of bright patches on the keogram reveals the main pulsations of the arc intensity, and vertical strips inside the patches are the signature of quicker scintillation

the auroral emissions. The sets of the WMI cameras mounted in Kiruna and Tjautjas consist of four cameras, two monochromatic cameras with narrowband filters centered at wavelengths of 560.0 and $430.0 \mathrm{~nm}$, one camera with a wideband filter centered at a wavelength of $675.0 \mathrm{~nm}$, and one color camera. Unfortunately, the 560.0 and 675.0 images were oversaturated during the event. The $430.0 \mathrm{~nm}$ camera takes one image with an exposure time of $4.27 \mathrm{~s}$ that cannot reproduce the peculiarities of rapidly changing auroral pulsations. For these reasons, for tomography-like reconstruction, we used the images of the color camera. According to the paper by Partamies et al. (2007), the main input in the green channel of the color camera comes from the auroral $557.7 \mathrm{~nm}$ emission. Thus, we used the green channel images for the tomography-like reconstruction of the volume distribution of the auroral emission, and hereinafter, we refer to this as the $557.7 \mathrm{~nm}$ emission distribution.

The three-dimensional structures of the auroral volume emission rates have been estimated over a horizontal region of $168 \times 130 \mathrm{~km}$, with an origin in Kiruna, between 80 and $180 \mathrm{~km}$ in height. This is done with a tomography-like algorithm developed by Björn Gustavsson (now at the Arctic University of Norway), especially for processing the data of the Auroral Large Imaging System (ALIS) (Brändström 2003) operated by the Swedish Institute of Space Physics. The algorithm uses a simultaneous iterative reconstruction technique and was described in detail in Gustavsson (1998).

When possible, we also performed a comparative analysis of a few $\mathrm{Hz}$ luminosity variations (internal modulation) in two neighboring pulsating arcs during the "on" phase of the pulsations. For the high-speed camera images, the mean intensities of certain $3 \times 3$ pixel areas of the pulsating auroral structures were calculated. Note that the result should not depend on the position of these areas along the arc (Sato et al. 2004).

\section{Observations}

\section{Geomagnetic and auroral background}

The observations were made at the beginning of the recovery phase of a moderate $(\sim 500 \mathrm{nT})$ magnetospheric substorm starting at 00:45 UT on March 15, 2018, when optical instruments at KRN and TJA were in the local postmidnight sector of magnetic local time. The aurora situation corresponded to a typical PsA event (e.g., Grono and Donovan 2020). We focus on the 45-s interval when a series of pulsating arc-like forms (hereinafter called pulsating arcs) were in the common part of the Kiruna and Tjautjas camera field of view with the most equatorial one near the local zenith of Tjautjas (Fig. 1a). The keogram in Fig. 1b shows seven on/off events in the near-zenith arc with a repetition period of a few seconds (main pulsations) and a few $\mathrm{Hz}$ scintillation during the "on" phase of the pulsations.

Figure 2 presents the horizontal distribution of the volume emission rate at an altitude of $95 \mathrm{~km}$. This distribution is the result of a tomography-like reconstruction of the 3D distribution of the $557.7 \mathrm{~nm}$ auroral emission for the time instance of 01:16:09 UT. The arc under consideration is indicated by a black arrow. The black line shows the position of the vertical slice of the volume emission rates chosen for the analyses. 


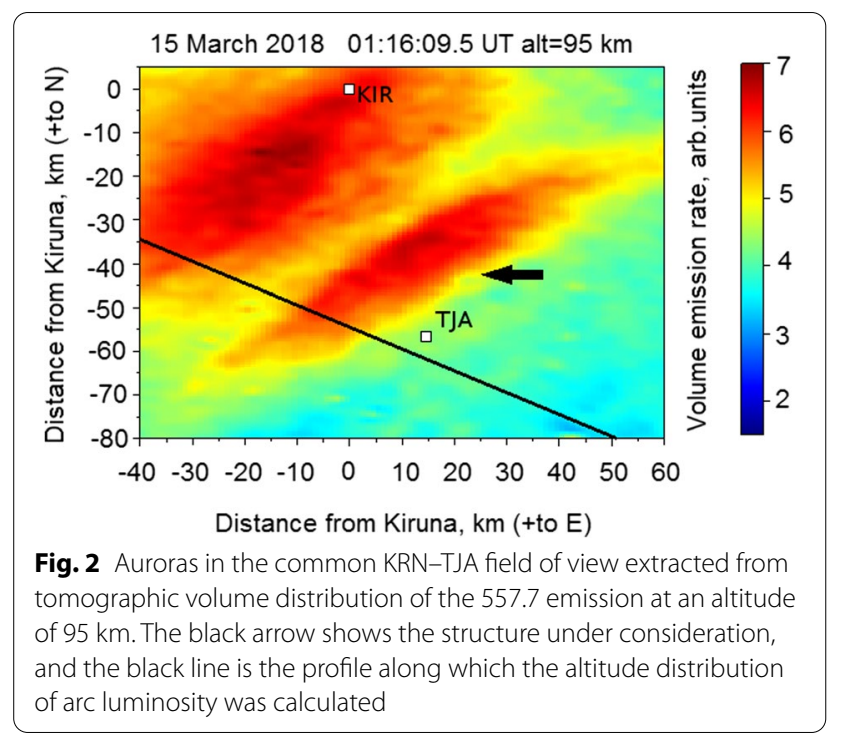

\section{Response of arc altitude to arc brightening}

Another result of tomographic reconstruction is shown in Fig. 3 as an altitude vs time diagram. Each vertical line in this diagram marks the altitude profile of the $557.7 \mathrm{~nm}$ volume emission rate at the horizontal position corresponding to where the black line crosses the arc of interest in Fig. 2, taken from the tomography result for every

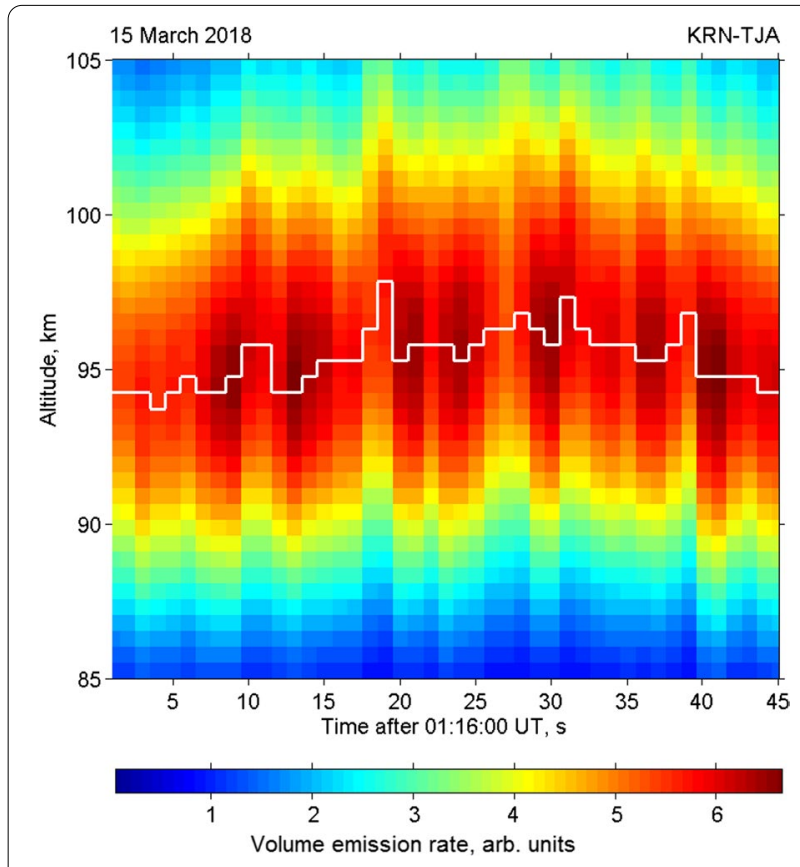

Fig. 3 Altitude vs time diagram taken in the vertical plane passing through part of the profile in Fig. 2 containing the arc examined. The white step-like line shows the time variation in the altitude of maximum luminosity second. The time interval corresponds to that for the keogram in Fig. 1b.

In general, the altitude of the volume emission rate maximum is approximately $95 \mathrm{~km}$, which corresponds to a characteristic energy of precipitating electrons of approximately $40 \mathrm{keV}$. This result is consistent with previous observations (e.g., Brown et al. 1976; Lessard 2012; Nishimura et al. 2020 and references therein). The temporal behavior of the altitude of the emission maximum (a white step-like line) shows the altitude of the more intense arc. In Fig. 4, the blue and red solid lines represent the altitude profiles of the volume emission rates of the $557.7 \mathrm{~nm}$ emission corresponding to times 01:16:11 UT and 01:16:14 in Fig. 3, respectively. The blue and red dashed lines are the results of calculations based on the auroral $557.7 \mathrm{~nm}$ emission model described in detail in Ivanov et al. (1993). The calculations were performed for isotropic Gaussian fluxes of precipitating electrons with characteristic energies of 41 and $44 \mathrm{keV}$.

\section{Features of internal modulation in two neighboring arcs}

An example of tomographic reconstruction of the arc under consideration is presented in Fig. 5. At the time of maximum intensity, the auroral structure in the TJA-R all-sky image looks like a single arc (Fig. 5a), whereas its tomographic reconstruction (Fig. 5b) indicates the presence of two arcs ( $\operatorname{arc} 1$ and $\operatorname{arc} 2$ ) inside the structure. Careful analysis of TJA-R images showed that the double structure of the arc might be detected without special

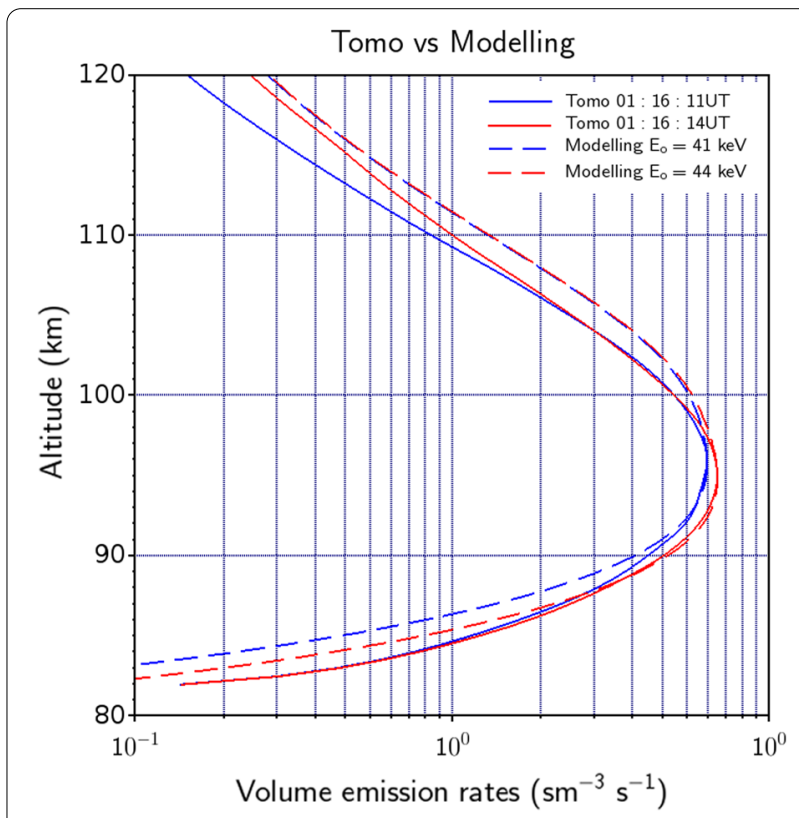

Fig. 4 The 557.7 reconstructed (solid line) and calculated (dashed line) emission rate profiles 


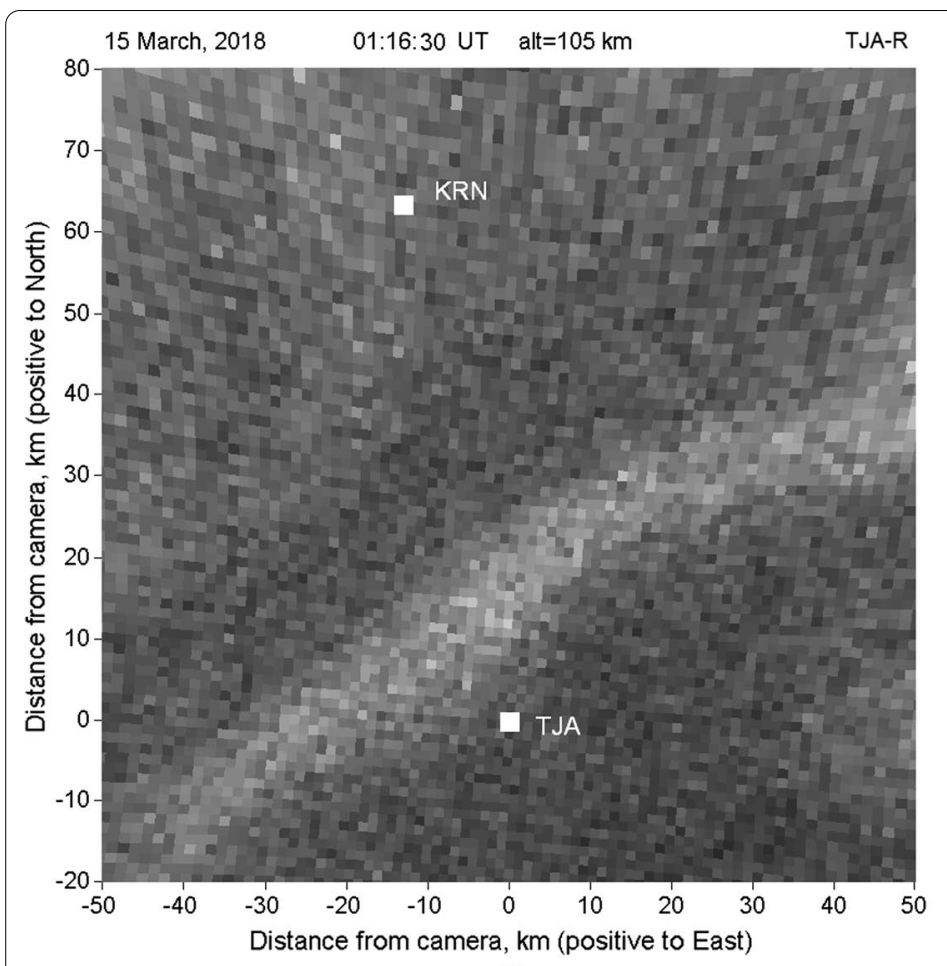

a

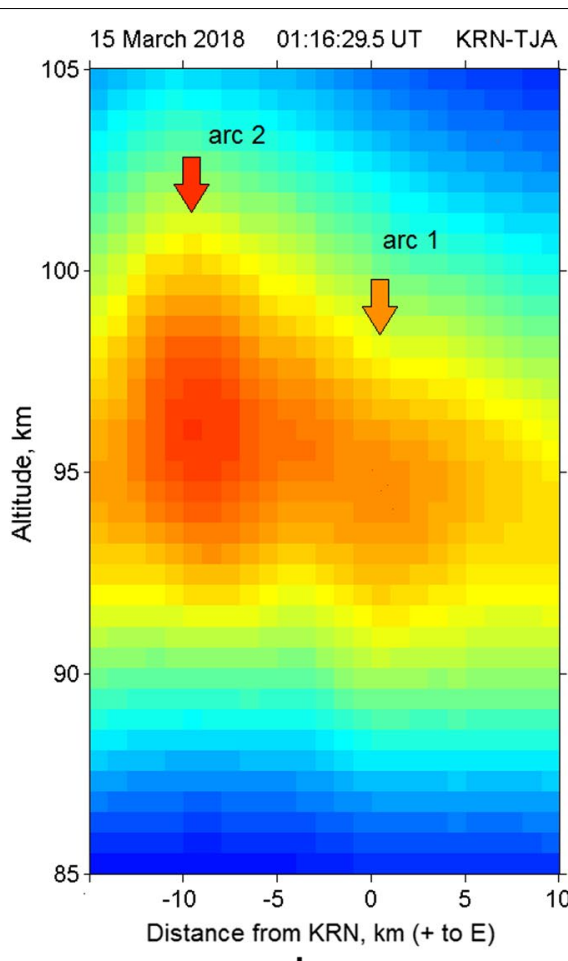

b

Fig. 5 a Auroral arc shape and location inferred from the TJA-R image; $\mathbf{b}$ tomographic reconstruction of the arc showing its multiple structures

processing for the first two pulses. Figure 6a shows an enlarged image of the auroral structure in the $60 \times 45$ pixel rectangular area near the zenith of TJA. For the moment presented, the two arcs are located very close to each other, giving an impression of a single wide structure in Fig. 1a. The impression is due to the way all-sky imaging hides one arc of the neighboring pair by the luminosity along the magnetic field line. The real cross section of the luminosity volume is seen only on images in the magnetic zenith, which is not the case in Fig. 1a. Due to the gap between these thin arcs on the frames, we were able to calculate the intensity variations in each arc averaged over a square area of $3 \times 3$ pixels, as well as for the neighboring background (Fig. 6b). The pulse with 3-s duration corresponding to the main pulsation is seen well in both arcs, but it is absent in the diffuse background. Internal modulation is also not detected in the background, which means that both main pulsations and internal modulation represent localized phenomena associated with the arcs only.

The more surprising finding is that only one arc (arc 1) demonstrates internal modulation. The modulation consists of an irregular sequence of intensity peaks at a repetition period of $\sim 0.4 \mathrm{~s}$, corresponding to a frequency of $2.5 \mathrm{~Hz}$. Tomography-like reconstruction also allowed us to recognize both arcs. In Fig. 6c they are marked with black arrows. Noticeably, within the switch-on interval, the arc without internal modulation (arc 2) is located higher (at altitude $\sim 95 \mathrm{~km}$ ) than $\operatorname{arc} 1$ with internal modulation $(\sim 93 \mathrm{~km})$.

A similar investigation was possible for the second switch-on interval, which still allowed us to distinguish the double structure of the pulsating arc on tomographic images (Fig. $7 \mathrm{~b}$ ). The results are the same: the pulse of main pulsations is manifested in both arcs but is not seen in the background luminosity (Fig. 7a); internal modulation occurs only in the arc 1 (upper panel in Fig. 7a), which is located at a lower altitude than the arc 2 without internal modulation (Fig. 7b). In both cases, the internal modulation consists of an irregular sequence of intensity peaks at a repetition period of $\sim 0.4 \mathrm{~s}$, corresponding to frequency of $2.5 \mathrm{~Hz}$.

Further development of auroral activity led to the two thin arcs merging in the all-sky images. A comparative analysis of the luminosity variations in the arcs therefore became impossible. However, the multiple structures of pulsating auroral form near the zenith could still be recognized on tomographic reconstructions. 


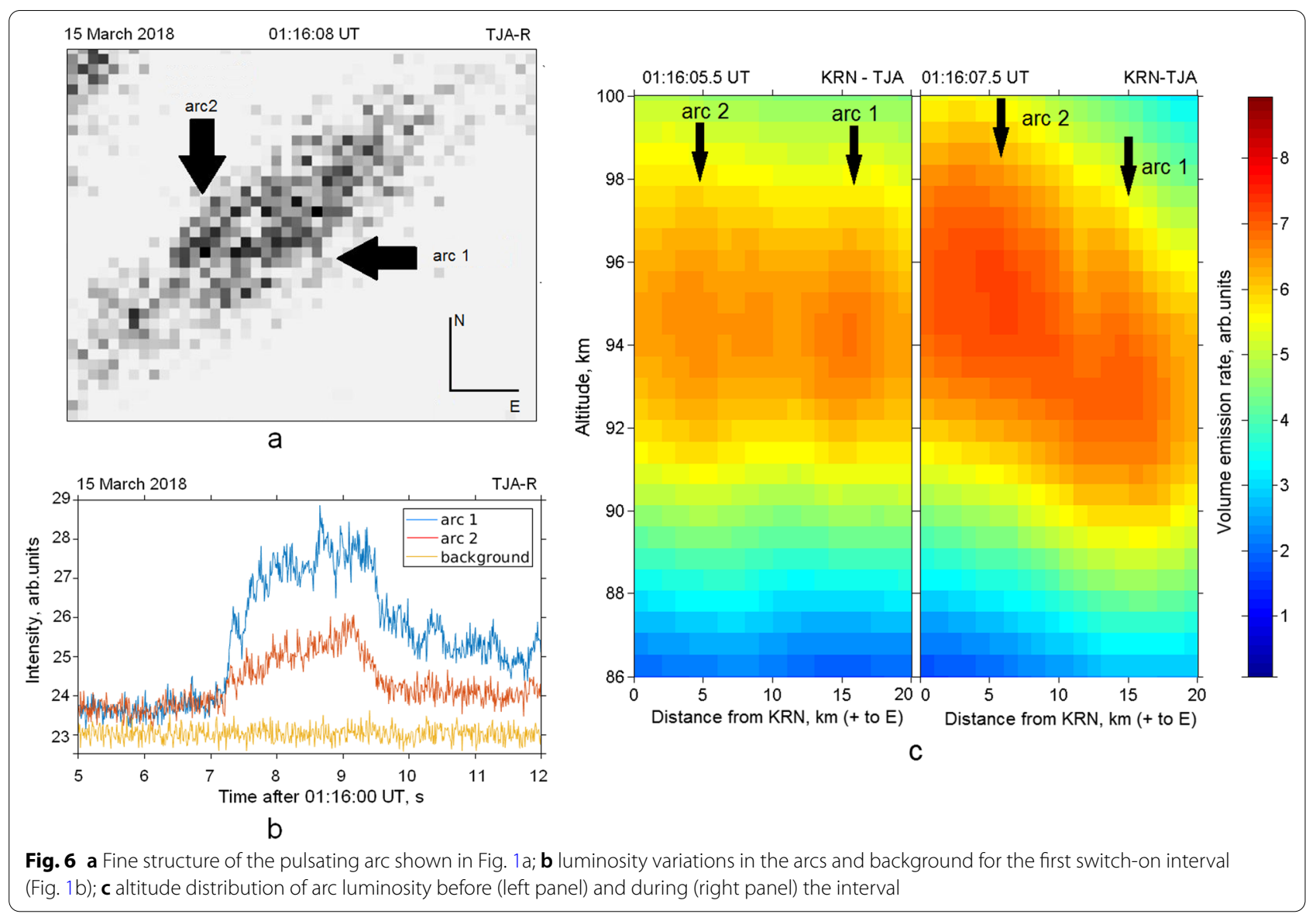

\section{Discussion}

\section{Generalization of the results}

In this section, we generalize the results and emphasize some details, which are useful for result interpretation.

We analyzed a series of seven on/off events that represent a typical PsA of an arc-like auroral structure demonstrating both main (a few seconds periodicity) and internal (a few Hz) modulations of luminosity. Two cameras (KRN and TJA) were located approximately along the meridian at a separation $\sim 60 \mathrm{~km}$ and provided allsky images of auroras with 1-s resolution. The arc was located in the KRN-TJA common field of view. This location allowed us to make a tomographic reconstruction of the altitude profile of arc luminosity so as to investigate the evolution of arc altitude over the course of the main pulsation. The third all-sky camera (TJA-R) provided the data at a higher time resolution (100 frames per second), and we used these data to study the internal modulation.

Intensity variations in the arc, averaged over squares of $10 \times 10$ pix, are shown in Fig. 8 . We put the square at the place where the arc is crossed by the black profile in Fig. 2. While the few-second variations look like quasi-sinusoidal oscillations, the internal modulation represents a rather irregular sequence of peaks with different time intervals between them. The mean value of the time interval, $T$, corresponds to frequency $f=1 / T \sim 2 \mathrm{~Hz}$. This is consistent with the result of a statistical investigation by Nishiyama et al. (2014), who showed that the occurrence probability of rapid modulation is localized between 2.0 and $2.5 \mathrm{~Hz}$.

Fast scintillations are observed only during the "on" phase of the main pulsations. Fig. 8 shows the sequence of peaks, corresponding to a few $\mathrm{Hz}$ modulation, which is highlighted in gray. There was no modulation in the background luminosity (before 01:16:07 UT) or between the pulses except at approximately 01:16:22 UT, when the next pulse of the main pulsation started just after the previous pulse (see, as well, Fig. 3). These facts are important for a possible explanation of fast scintillations, which will be discussed in the next section.

The altitude of the arc changes. Regarding the examined arc as a homogeneous structure, we have found the following features of its behavior (see Fig. 3). The "arc altitude", defined by us as the position of the luminosity maximum, was lower within the switch-on intervals than between them. The altitude changes by $1.5-3 \mathrm{~km}$, which 


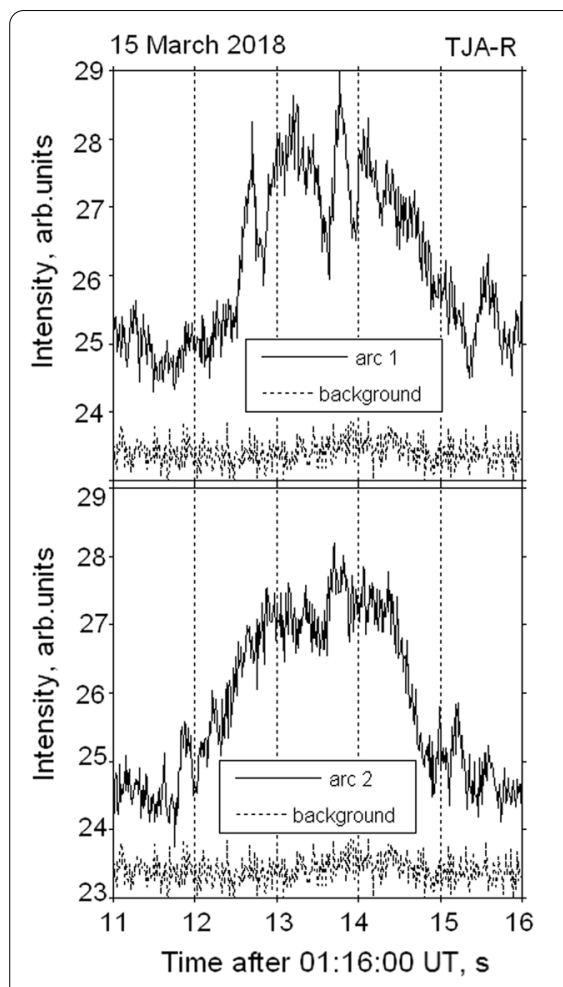

a

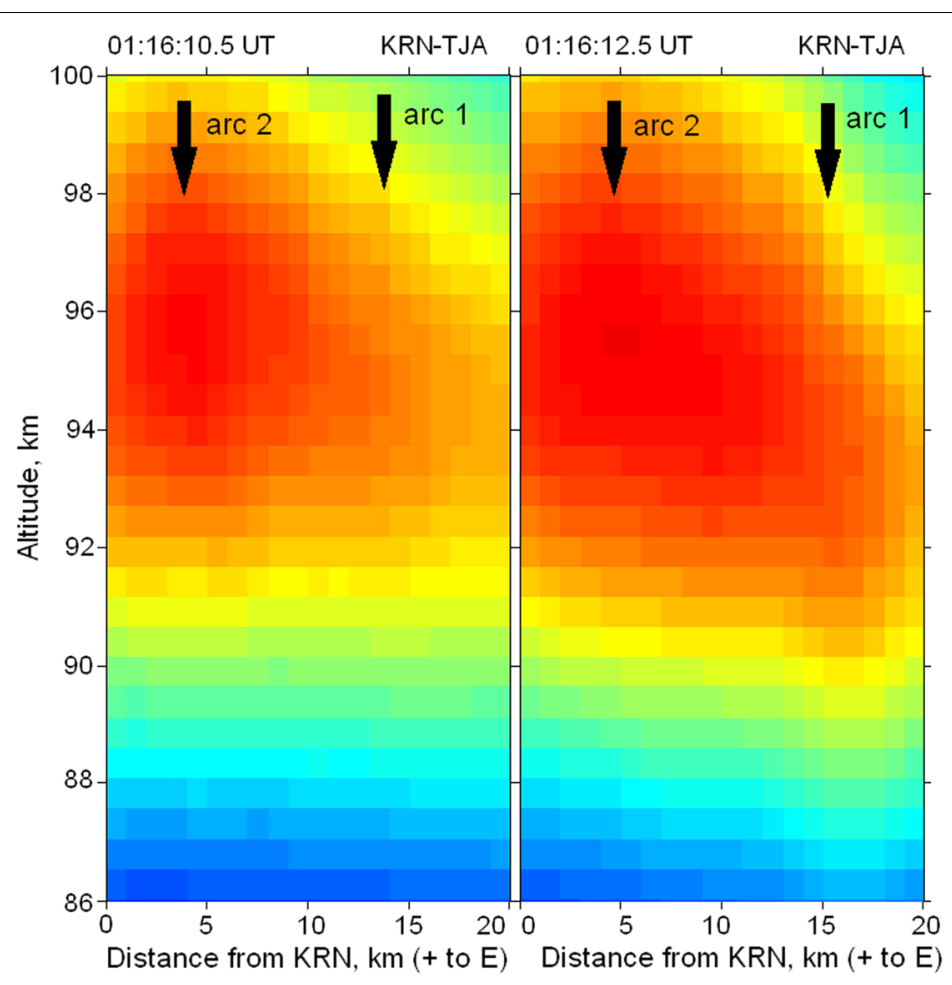

b

Fig. 7 a Luminosity variations in the arcs and background for the second switch-on interval (Fig. 1b); b altitude distribution of arc luminosity before (left panel) and during (right panel) the interval

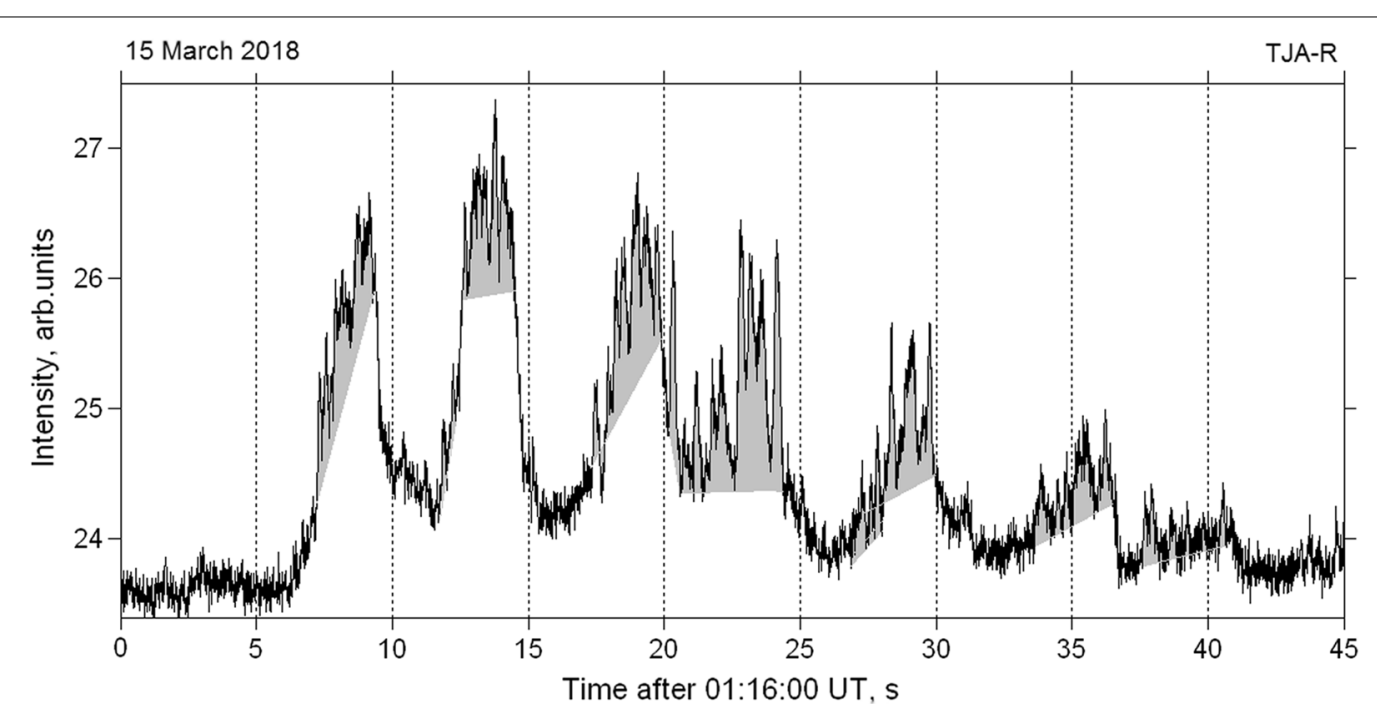

Fig. 8 Luminosity variation in the examined arc averaged over the square $10 \times 10$ pix. The internal modulation is highlighted with gray

is larger than the spatial resolution of the optical tomography method $(\sim 500 \mathrm{~m})$. To the authors' knowledge, earlier observations of PsA showed no indication of changes in the height of the lower border during the lifetime of a single pulsation (e.g., Brown et al. 1976). This is probably due to the lower spatial resolution of the triangulation 
method, which is traditionally used in such investigations. The decrease in arc altitude means that the energy of precipitating particles increases.

Of the two closely spaced arcs, internal modulation took place only in the lowest arc. In addition to the high spatial resolution (in comparison with triangulation), one more merit of optical tomography is its ability to distinguish the fine structure of the auroral arc, whereas on ordinary all-sky images, this may be masked by neighboring structures (see Fig. 5). A more careful analysis of the original frames of the rapid camera TJA-R showed that the structure considered actually consists of two thin arcs, at least at the beginning of the interval (Fig. 6a). This allowed us to calculate the variation in luminosity in each arc separately (Figs. 6, 7). The comparison with tomographic reconstructions showed that internal modulation appears in the arc which has lower altitude during the "on" phase. One can say that the result is consistent with the early study by Royrvik and Davis (1977), who showed that in many cases, $3 \pm 1 \mathrm{~Hz}$ modulation is detected only near the form boundary. Later, Whiter et al. (2010) found that the energy of flickering electron precipitation was higher than the energy of non-flickering auroral electrons.

\section{Two approaches for interpretation of PsA features: pitch-angle scattering}

One widely accepted primary process for PsA is pitchangle scattering of electrons in the magnetosphere ( $\mathrm{Li}$ et al. 2012, and references therein). Another process was proposed by Sato et al. (2004), who showed that time variations of the field-aligned potential drop may cause the pulsating aurora. In what follows, for brevity, we will refer to these approaches as scattering and accelerating approaches.

Both approaches are based on satellite measurements, and the generation mechanism of some PsA features is still not fully understood due to a lack of in situ observations. Indeed, cases of successful location of satellites with respect to PsA patterns are very rare events by themselves, and not all such events allow the correct association of satellite measurements with optical pulsations in the ionosphere. To reduce the uncertainty in locating a satellite's magnetic footprint, special methods should be applied (e.g., Hosokawa et al. 2020). Because of this, we do not give preference to any of the above approaches and show below that our results regarding the brightening pulsating arc can be explained in the frame of both approaches.

We have shown that the altitude of the emission is lower during the "on" phases of PsA, which means that the energy of precipitating electrons is higher at these moments. In the frame of the scattering approach, the result can be explained by the model of Miyoshi et al.
(2015). They demonstrated that PsA during the "on" phase is caused by scattering of electrons due to the lower/upper band chorus. The upper (lower) band resonates with lower (higher) energy electrons. Both higherand lower-energy electrons are detected during the "on" phase, whereas only lower-energy electron precipitation, caused by the upper band, is seen during the OFF phase. Such precipitation causes relatively higher-altitude emissions. One other possible explanation is that variations in cold plasma density that potentially correspond to the on-off pulsations of the pulsating aurora would change the resonant energy of chorus-electron scattering (e.g., $\mathrm{Li}$ et al. 2012).

The internal modulation may also be explained in the frame of the scattering approach. Hosokawa et al. (2020) demonstrated a direct association between the multiscale temporal variations in chorus wave power detected onboard the Arase satellite and aurora luminosity inferred from ground optical measurements. Namely, they showed correlations between chorus bursts and the main optical pulsations, as well as between discrete chorus elements embedded in a burst and internal modulation embedded in an impulse of main pulsations (a Matryoshka doll configuration).

Hosokawa et al. (2020) noted that the energy of precipitating electrons responsible for pulsating auroras often ranges from a few to several tens of $\mathrm{keV}$. Due to different time-of-flight from the magnetosphere to the ionosphere, significant spreading in time of the electron flux appears. In such a case, the sub-second modulation in the chorus tends to be smeared out, and ground-based optical instruments do not see the corresponding internal modulation in the PsA emission. If the resonance energy is higher (in our case, this means that the corresponding auroral arc has lower altitude), the dispersion effect would be smaller, and sub-second (internal) modulation would be able to survive in the optical data.

\section{Periodic acceleration as a possible mechanism for internal modulation}

In this section, we present a simple physical model that explains all three of our findings - the decrease in the arc altitude in the course of its brightening, internal modulations inside the brightening arc, and absence of internal modulation in the arc located higher than the arc with sub-second pulsations.

By using the Swarm satellite, Gillies et al. (2015) identified upward field-aligned current (FAC) throughout the interior of the PsA (pulsating patches). It is widely assumed that aurora brightening is due to an increase in the flux of precipitating particles, which may mean an increase in FAC. In the course of its increase, the FAC can exceed the threshold for electrostatic ion-cyclotron 
instability (EIC instability) resulting in anomalous resistivity and yielding a field-aligned potential drop (e.g., Papadopoulos 1977). The potential drop accelerates the precipitating electrons along the magnetic field line and the altitude of the corresponding arc decreases during the "on" phase. Signatures of electron acceleration were found in FAST satellite data by Sato et al. (2004). We leave the reason for current enhancement at the beginning of each switch-on pulse of main pulsations as beyond the scope of our study and consider the further possible evolution of the current during the "on" phase.

Safargaleev (1996) showed that the value of the EIC instability threshold inside the anomalous resistivity area decreases due to a change in some plasma parameters in the course of instability development. After the appearance of the anomalous resistivity, the FAC should decrease following Ohm's law. When the FAC becomes less than the new threshold, then the anomalous resistivity and potential drop turn off, the FAC starts to increase again, and the on/off process becomes quasi-periodic. Earlier, the idea of excitation of periodic events in a circuit with a double layer was suggested by Alfven (1981). Later, quasi-periodic oscillation of anomalous resistivity was discussed by Pilipenko et al. (1999) as an additional possible mechanism for the generation of geomagnetic pulsations in the few $\mathrm{Hz}$ range. In our case, the periodic switch on/off of a fieldaligned potential drop may lead to oscillation of the flux of precipitating particles either directly or via the periodic launch of VLF waves, which then periodically scatter the electrons into the loss cone (Liatskii and Safargaleev 1985).

The stabilization time of the FAC inside the layer of anomalous resistivity is determined by the parameters of the layer. Alfven waves are one of the basic mechanisms for energy and current transport in near-Earth plasmas. Connecting the stabilization of the current with the propagation of an Alfvén wave across the layer, for a rough estimation of the pulsation period, $T$, we can put $T \sim 2 b / V_{A}$. Here, $V_{A}$ is the Alfvén velocity at the place of anomalous resistivity development, and $b$ is the width of the layer along the magnetic field line. Although the expression is very simple, both parameters are unknown. Let us assume that $V_{A}$ does not change dramatically along the magnetic field line and, according to Kim et al. (2018), reaches a value of $\sim 1500 \mathrm{~km} / \mathrm{s}$ in the postmidnight sector where our observations occur. Then, to obtain the frequency of potential drop oscillations $\sim 2 \mathrm{~Hz}$, one needs to take the other unknown parameter $b$ to be approximately $400 \mathrm{~km}$. To the author's knowledge, only the dimension along the meridian (i.e., across the magnetic field lines) is known for areas where field-aligned acceleration presumably took place (Torbert and Mozer 1978). In particular, the authors noted that the geometry of accelerating structures is similar to that of auroral arcs.
The absence of internal modulation in the upper arc (arc 2 in Figs. 5, 6) may simply mean that the arc-related field-aligned current does not achieve the threshold value due to, for example, different conditions for the development of EIC instability above the arcs.

In many papers related to pulsating auroras, there is no direct indication of exactly what auroral form is under consideration, although the generation mechanisms for pulsating patches and pulsating arcs may be different. In accordance with Sato et al. (2015), applying the fieldaligned electric field modulation model to elongated pulsating arcs may be more reasonable than the pitch-angle scattering approach if one takes into account the analogy to ordinary auroral arcs. Ordinary arcs are enhanced via field-aligned electric field accelerations, as is widely accepted due to in situ observations (e.g., Torbert and Mozer 1978).

\section{Conclusion}

Data from three all-sky cameras in Kiruna and Tjautjas (Sweden) were used to estimate the altitude of pulsating arc-like forms by means of optical tomography. The event occurred during substorm recovery phase and represented both periodic switch on/off luminosity of the arc with a repetition period of a few seconds (main pulsations) and faster scintillation (approximately $2 \mathrm{~Hz}$ ) observed during the "on" phase of the main pulsations. The main results of investigations are following:

1. The main pulsations and internal modulation are localized phenomena confined to the arcs.

2. The altitude of the arc during the "on" phases is $\sim 93 \mathrm{~km}$, which is $1-2 \mathrm{~km}$ lower than that for the "off" phases.

3. Internal modulation of luminosity takes place only in the lowest arc.

4. The results may be interpreted in the frame of the traditionally accepted mechanism assuming electron scattering via the VLF-wave/particle interaction in the equatorial magnetosphere, while the internal modulation may also be alternatively interpreted in the frame of the (less-often suggested) mechanism of field-aligned acceleration somewhere between the equatorial plane and ionosphere.

\footnotetext{
Abbreviations

ALIS: Auroral Large Imaging System; ECH: Electrostatic electron cyclotron harmonic; EIC instability: Electrostatic ion-cyclotron instability; EMCCD: Electron multiplying charge-coupled device; FAC: Field-aligned current; FAST: Fast Auroral Snapshot Explorer; LBH: Lower-band chorus and electrostatic electron cyclotron harmonic; PsA: Pulsating auroras; $V_{A}$ : Alfven velocity; VLF: Very low frequency.
} 


\section{Acknowledgements}

The work of Japanese co-authors has been supported by JSPS KAKENHI JP $15 \mathrm{H} 05747$ and $16 \mathrm{H} 06286$. The operation of the EMCCD camera at Tjautjas has been supported by Swedish Institute of Space Physics. We acknowledge support through the Swedish National Space Agency (SNSA) to the Swedish Institute of Space Physics Dnr.153/16

\section{Authors' contributions}

VS performed the data analysis and draft the manuscript. TS performed the optical tomography and contributed to the draft of manuscript. YO and $\mathrm{KH}$ carried out the optical observation and processed the data. SO, YM, SK and RF discussed the interpretation of the observation. All authors read and approved the final manuscript.

\section{Funding}

This work has been supported by JSPS KAKENHI 15H05747, JSPS KAKENHI $16 \mathrm{H} 06286$

\section{Availability of data and materials}

The EMCCD all-sky camera data from Tjautjas are accessible at the following https://doi.org/10.34515/DATA.GND-0062-0006-0204_v01 The watec monochromatic/color data are accessible at the following links:

http://pc115.seg20.nipr.ac.jp/www/optical/watec/krn/rawdata/ (for Kiruna data)

http://pc115.seg20.nipr.ac.jp/www/optical/watec/tja/rawdata/ (for Tjautjas data)

They are also connected to the AQVN database:

http://pc115.seg20.nipr.ac.jp/www/AQVN/index.html.

\section{Declarations}

Ethics approval and consent to participate

Not applicable.

\section{Consent for publication}

Not applicable.

\section{Competing interests}

The authors declare that they have no competing interests.

\section{Author details}

${ }^{1}$ Polar Geophysical Institute RAS, Apatity, Russia. ${ }^{2}$ Swedish Institute of Space Physics, Kuruna, Sweden. ${ }^{3}$ University of Electro-Communications, Tokyo, Japan. ${ }^{4}$ Institute for Space-Earth Environmental Research, Nagoya University, Nagoya, Japan. ${ }^{5}$ National Institute of Polar Research, Tokyo, Japan. ${ }^{6}$ Space Physics and Astronomy Research Unit, University of Oulu, Oulu, Finland. ${ }^{7}$ The Graduate University for Advanced Studies, SOKENDAI, Tokyo, Japan. ${ }^{8}$ Joint Support-Center for Data Science Research, Research Organization of Information and Systems, Tokyo, Japan. ${ }^{9}$ Research Institute for Sustainable Humanosphere, Kyoto University, Kyoto, Japan. ${ }^{10}$ Research Organization of Information and Systems, Toranomon, Minato-ku, Tokyo, Japan.

Received: 10 November 2021 Accepted: 6 February 2022

Published online: 18 February 2022

\section{References}

Alfven H (1981) Electric currents in space plasma. In: Blamont JE et al (eds) Cosmic plasma, series Astrophysics and Space Science Library, vol 82. Springer, Berlin, pp 11-41

Arnoldy RL, Dragoon K, Cahill LJ, Mende SB, Rosenberg TJ (1982) Detailed correlations of magnetic field and riometer observations at $L=4.2$ with pulsating aurora. J Geophys Res 87:10449-10456. https://doi.org/10. 1029/ja087ia12p10449

Brändström BUE (2003) The auroral large imaging system-Design, operation and scientific results. Dissertation, Institutet för rymdfysik, Kiruna, Sweden.
Brown NB, Davis TN, Hallinan TJ, Stenbaek-Nielsen HC (1976) Altitude of pulsating aurora determined by a new instrumental technique. Geophys Res Lett 3:403-404. https://doi.org/10.1029/GL003i007p00403

Bryant D, Courtier G, Bennett G (1971) Equatorial modulation of electrons in a pulsating aurora. J Atm Terr Phys 33:859-867. https://doi.org/10.1016/ 0021-9169(71)90086-9

Fukizawa M, Sakanoi T, Miyoshi Y, Hosokawa K, Katoh Y et al (2018) Electrostatic electron cyclotron harmonic waves as a candidate to cause pulsating aurora. Geophys Res Lett 45:661-668. https://doi.org/10.1029/2018G L080145

Gillies DM, Knudsen D, Spanswick E, Donovan E, Burchill J, Patrick M (2015) Swarm observations of field-aligned currents associated with pulsating auroral patches. J Geophys Res 120:9484-9499. https://doi.org/10.1002/ 2015JA021416

Grono E, Donovan E (2020) Surveying Pulsating Auroras. Ann Geophys 38:1-8. https://doi.org/10.5194/angeo-38-1-2020

Gustavsson B (1998) Tomographic inversion for ALIS noise and resolution. J Geophys Res 103:26621-26632. https://doi.org/10.1029/98JA00678

Hosokawa K, Ogawa Y, Kadokura A, Miyaoka H, Sato N (2010) Modulation of ionospheric conductance and electric field associated with pulsating aurora. J Geophys Res. https://doi.org/10.1029/2009JA014683

Hosokawa K, Miyoshi Y, Ozaki M et al (2020) Multiple time-scale beats in aurora: precise orchestration via magnetospheric chorus waves. Sci Rep. https://doi.org/10.1038/s41598-020-59642-8

Ivanov VE, Kirillov AS, Sergienko TI, Steen A (1993) Modeling of the altitude distribution of green line $(\lambda 5577 \mathrm{~A})$ luminosity in aurora. In SPIE. https:// doi.org/10.1117/12.164815

Johnstone AD (1978) Pulsating aurora. Nature 274:119-126. https://doi.org/10. 1038/274119a0

Kataoka R, Fukuda Y, Uchida HA, Yamada H, MiyoshiY EY, Hampton D (2016) High-speed stereoscopy of aurora. Ann Geophys 34:41-44. https://doi. org/10.5194/angeo-34-41-2016

Kim K-H, Kim G-J, Kwon H-J (2018) Distribution of equatorial Alfvén velocity in the magnetosphere: a statistical analysis of THEMIS observations. Earth Planets Space 70:174. https://doi.org/10.1186/s40623-018-0947-9

Lessard MR (2012) A review of Pulsating Aurora. In: Keiling A et al (eds) Auroral Phenomenology and Magnetospheric Processes: Earth and Other Planets, vol 197. AGU, Washington, pp 55-68

Li W, Bortnik J, Nishimura Y, Thorne RM, Angelopoulos V (2012) The origin of pulsating aurora: Modulated whistler mode chorus. In: Keiling A (ed) Auroral Phenomenology and Magnetospheric Processes, vol 197. AGU, Washington, pp 379-388

Liatskii VB, Safargaleev WV (1985) Generation of VLF waves in a region of anomalous resistance. Geomagn Aeronomy 25:958-961 ((in Russian))

McEwen DJ, Yee E, Whalen BA, Yau AW (1981) Electron energy measurements in pulsating auroras. Can J Phys 59:1106-1115. https://doi.org/10.1139/ p81-146

Miyoshi Y, Saito S, Seki K et al (2015) Relation between fine structure of energy spectra for pulsating aurora electrons and frequency spectra of whistler mode chorus waves. J Geophys Res 120:7728-7736. https://doi.org/10. 1002/2015JA02156

Nishimura Y, Lessard MR, Katoh Y et al (2020) Diffuse and Pulsating Aurora. Space Sci Rev 216:357-387. https://doi.org/10.1007/s11214-019-0629-3

Nishiyama T, Sakanoi T, Miyoshi Y, Hampton DL, Katoh Y, Kataoka R, Okano S (2014) Multiscale temporal variations of pulsating auroras: On-off pulsation and a few Hz modulation. J Geophys Res 119:3514-3527. https://doi. org/10.1002/2014JA019818

Ogawa Y, Tanaka Y, Kadokura A, Hosokawa K et al (2020) Development of lowcost multiwavelength imager system for studies of aurora and airglow. Polar Sci. https://doi.org/10.1016/j.polar.2019.100501

Papadopoulos K (1977) A review of an anomalous resistivity for the ionosphere. Rev Geophys Space Phys 15:113-127. https://doi.org/10.1029/ RG015i001p00113

Partamies N, Syrjäsuo M, Donovan E (2007) Using color in auroral imaging. Can J Phys 85:101-109. https://doi.org/10.1139/p06-090

Pilipenko VA, Shalimov SL, Fedorov EN, Engebretson MJ, Hughes WJ (1999) Coupling between field-aligned current impulses and Pi1 noise bursts. J Geophys Res 104:17419-17430. https://doi.org/10.1029/1999JA900190

Royrvik O, Davis TN (1977) Pulsating aurora: local and global morphology. J Geophys Res 82:4720-4740. https://doi.org/10.1029/JA082i029p04720 
Safargaleev VV (1996) Disturbances in the magnetosphere-ionosphere system. Dissertation, Leningrad State University

Sandahl I, Eliasson L, Lundin R (1980) Rocket observations of precipitating electrons over a pulsating aurora. Geophys Res Lett 7:309-312. https:// doi.org/10.1029/GL007i005p00309

Sato N, Wright DM, Carlson CW, Ebihara Y, Sato M, Saemundsson T, Milan S, Lester M (2004) Generation region of pulsating aurora obtained simultaneously by the FAST satellite and a Syowa-lceland conjugate pair of observatories. J Geophys Res. https://doi.org/10.1029/2004JA010419

Sato N, Kadokura A, Tanaka Y, Nishiyama T, Hori T, Yukimatu AS (2015) Omega band pulsating auroras observed onboard THEMIS spacecraft and on the ground. J Geophys Res 120:5524-5544. https://doi.org/10.1002/2015 J A021382

Shiokawa K, Katoh Y, Hamaguchi Y, Yamamoto Y, Adachi T, Ozaki M, Oyama S-I, Nosé M et al (2017) Ground-based instruments of the PWING project to investigate dynamics of the inner magnetosphere at subauroral latitudes as a part of the ERG-ground coordinated observation network. Earth Planets Space 69:160. https://doi.org/10.1186/s40623-017-0745-9

Tesema F, Partamies N, Tyssøy HN, Kero A, Smith-Johnsen C (2020) Observations of electron precipitation during pulsating aurora and its chemical impact. J Geophys Res 125:e2019. https://doi.org/10.1029/2019JA027713 Torbert RB, Mozer FS (1978) Electrostatic shocks as the source of discrete auroral arcs. Geophys Res Lett 5:135-138. https://doi.org/10.1029/GL005 i002p00135

Whiter DK, Gustavsson BS, IvchenkoDahlgren BH (2010) Using multispectral optical observations to identify the acceleration mechanism responsible for flickering aurora. J Geophys Res. https://doi.org/10.1029/2010JA0158 05

\section{Publisher's Note}

Springer Nature remains neutral with regard to jurisdictional claims in published maps and institutional affiliations.

\section{Submit your manuscript to a SpringerOpen ${ }^{\circ}$ journal and benefit from:}

- Convenient online submission

- Rigorous peer review

- Open access: articles freely available online

- High visibility within the field

- Retaining the copyright to your article

Submit your next manuscript at $\boldsymbol{\nabla}$ springeropen.com 